\title{
Chemical composition of photospheres in RS CVn stars
}

\author{
G. Tautvaišienè,${ }^{1}$ G. Barisevičius,${ }^{1}$ S. Berdyugina,${ }^{2}$ I. Ilyin, ${ }^{3}$ \\ and Y. Chorniy ${ }^{1}$ \\ ${ }^{1}$ Institute of Theoretical Physics and Astronomy, Vilnius University, \\ Goštauto 12, LT-01108 Vilnius, Lithuania \\ email: grazina.tautvaisiene@tfai.vu.lt \\ ${ }^{2}$ Kiepenheuer Institut für Sonnenphysik, Schöneckstr 6, D-79104 Freiburg, Germany \\ email: sveta@kis.uni-freiburg.de \\ ${ }^{3}$ Astrophysikalisches Institut Potsdam, An der Sternwarte 16, D-14482 Potsdam, Germany \\ email: ilyin@aip.de
}

\begin{abstract}
In order to investigate how magnetic activity influences a stellar evolution, we undertake a detailed study of photospheric abundances in a sample of RS CVn stars. Using the Nordic Optical Telescope we investigate abundances of more than 20 chemical elements, including carbon isotopes, nitrogen and other mixing-sensitive species. In this presentation we report on current results of the analysis, among which there are first evidences that extramixing processes may start acting in low-mass chromospherically active stars below a bump of the luminosity function of red giants.
\end{abstract}

Keywords. Stars: abundances, stars: evolution, stars: magnetic fields

\section{Introduction}

RS CVn-type variables are remarkable due to large starspots, strong chromospheric plages, coronal X-ray and microwave emissions, as well as strong flares in the optical, radio and other spectral regions. The photometric brightness variation analysis, Doppler imaging and spectral line analysis of RS CVn stars indicate that starspots may cover up to about $40 \%$ of a stellar surface. The photospheric abundances of chemical elements in RS CVn-type stars are found to be peculiar and indicate a combined action of various physical processes related to activity. Peculiar elemental abundances were found in the coronal spectra of RS CVn stars as determined using cosmic satellites as well. In order to interpret the apparent abundance anomalies, we have started a detailed study of the photospheric abundances in about thirty RS CVn stars. A detailed study of photospheric abundances was already carried out for the following RS CVn stars: $\lambda$ And (Tautvaišiene et al. 2010), 29 Dra (Barisevičius et al. 2010), 33 Psc (Barisevičius et al. 2011), AY Cet (Tautvaišienè et al. 2011), AZ Psc, and 3 Cam (this work).

\section{Overview}

Low-mass start to modify their surface carbon and nitrogen abundances at a bottom of the red giant branch where they undergo the so-called first dredge-up Iben (1965). During this evolutionary stage, the deepening convective envelope mixes the outer layers of the red giant with the internal matter which has been $\mathrm{CN}$-processed while the star resided on the main sequence. Convective mixing induces a change of the carbon and nitrogen surface abundances $\left({ }^{12} \mathrm{C}\right.$ decrease, while the ${ }^{13} \mathrm{C}$ and ${ }^{14} \mathrm{~N}$ abundances increase). 

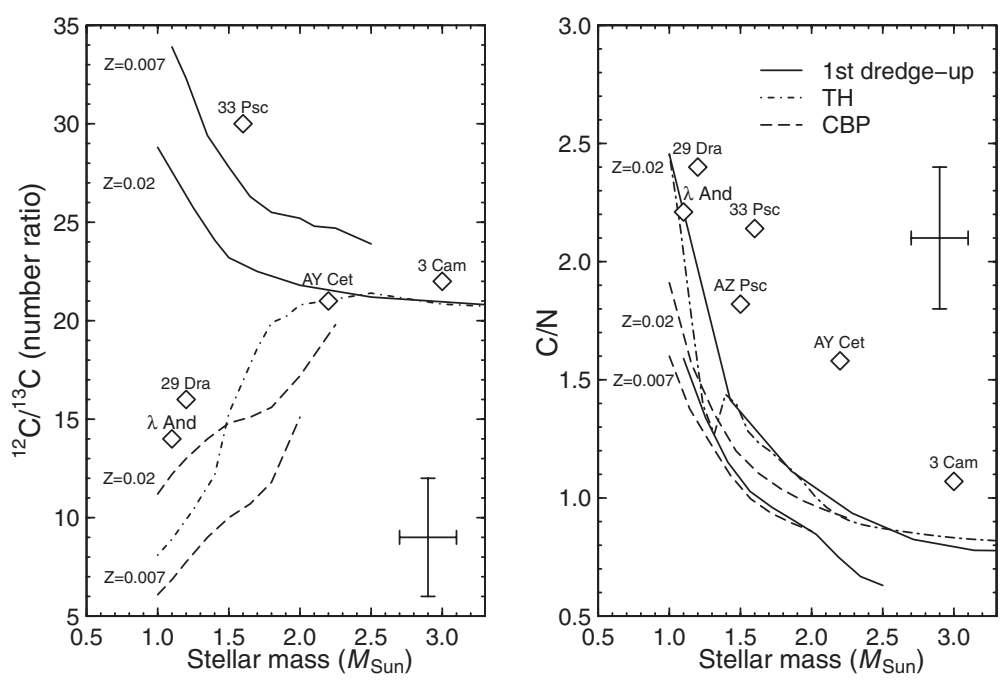

Figure 1. Comparisons of $\mathrm{C} / \mathrm{N}$ and ${ }^{12} \mathrm{C} /{ }^{13} \mathrm{C}$ ratios in the $\mathrm{RS} \mathrm{CVn}$ stars with the theoretical models as explained in the text.

In Fig. 1 we compare ${ }^{12} \mathrm{C} /{ }^{13} \mathrm{C}$ and $\mathrm{C} / \mathrm{N}$ ratios of the RS CVn stars investigated with the theoretical model of the first dredge-up and the current models of 'cool bottom processing' (CBP, Boothroyd \& Sackman 1999), and 'thermohaline mixing' (TH, Charbonnel \& Lagarde 2010).

The evolutionary sequences in the luminosity vs. effective temperature diagram by Girardi et al. (2000) show that all six investigated RS CVn stars are first ascent giants, lying below the red giant sequence bump. According to the standard theory, such stars should have carbon and nitrogen abundance alterations caused only by the first dredge-up. The extra mixing in low-mass stars starts acting only at the red giant branch luminosity bump.

In our sample, two low-mass active RS CVn stars $\lambda$ And and 29 Dra have ${ }^{12} \mathrm{C} /{ }^{13} \mathrm{C}$ ratios, which are lowered by extra mixing. They give a hint that extra-mixing processes may start acting in these low-mass chromospherically active stars slightly earlier than at the bump of the red giant sequence in non-active stars. The other low-mass star, 33 Psc, which has almost negligible activity, has the normal carbon isotope ratio as well as the other less evolved RS CVn stars of higher masses (AY Cet and $3 \mathrm{Cam}$ ). The ${ }^{12} \mathrm{C} /{ }^{13} \mathrm{C}$ ratio in AZ Psc was not determined. The $\mathrm{C} / \mathrm{N}$ ratios in almost all of the stars lie slightly above the theoretical model.

\section{References}

Iben, I., Jr. 1965, ApJ, 142, 1447

Barisevičius, G., Tautvaišienè, G., Berdyugina, S., Chorniy, Y., \& Ilyin, I. 2010, Baltic Astronomy, 19, 157

Barisevičius, G., Tautvaišienè, G., Berdyugina, S., Chorniy, Y., \& Ilyin, I. 2011, Baltic Astronomy, 20, 53

Boothroyd, A. I. \& Sackman, I. J. 1999, ApJ, 510, 232

Charbonnel, C. \& Lagarde, N. 2010, A\&A, 522, A10

Girardi, L., Bressan, A., Bertelli, G., \& Chiosi, C. 2000, A\& AS, 141, 371

Tautvaišienè, G., Barisevičius, G., Berdyugina, S., Chorniy, Y., \& Ilyin, I. 2010, Baltic Astronomy, 19, 95

Tautvaišienè, G., Barisevičius, G., Berdyugina, S., Chorniy, Y., \& Ilyin, I. 2011, AN, 332, 925 